\title{
miR-142-3p Modulates Cell Invasion and Migration via PKM2- Mediated Aerobic Glycolysis in Colorectal Cancer
}

\author{
JunYu Ren, ${ }^{1}$ Wenliang Li $\mathbb{D}^{1},{ }^{1}$ Guoqing Pan, ${ }^{2}$ Fengchang Huang, ${ }^{1}$ Jun Yang, \\ Hongbin Zhang, ${ }^{1}$ Ruize Zhou, ${ }^{1}$ and Ning $\mathrm{Xu}^{1}$ \\ ${ }^{1}$ Department of Oncological Surgery, First Affiliated Hospital of Kunming Medical University, Kunming, Yunnan, China \\ ${ }^{2}$ Department of Pathology, First Affiliated Hospital of Kunming Medical University, Kunming, Yunnan, China \\ Correspondence should be addressed to Wenliang Li; li_wl1@163.com
}

Received 9 March 2021; Revised 21 June 2021; Accepted 28 June 2021; Published 14 July 2021

Academic Editor: Elena Andreucci

Copyright (c) 2021 JunYu Ren et al. This is an open access article distributed under the Creative Commons Attribution License, which permits unrestricted use, distribution, and reproduction in any medium, provided the original work is properly cited.

\begin{abstract}
Decreased expression of miR-142-3p was observed in human cancers. However, the function and mechanism of miR-142-3p in human colorectal cancer remain obscure. The expressions of miR-142-3p in human colorectal cancer tissues and cell lines were measured by RT-qPCR. The effects of miR-142-3p on cell invasion and migration were detected by transwell assays. The efficiency of aerobic glycolysis was determined by glucose consumption and lactate production. Dual-luciferase reporter assays were performed to confirm the correlation between miR-142-3p and pyruvate kinase isozyme M2 (PKM2). The level of PKM2 was assessed by western blotting. Our results showed that the expression of miR-142-3p was decreased both in human colorectal cancer tissues and in cells. Overexpression of miR-142-3p in cell line attenuated colorectal cancer cell invasion and migration. About the underlying mechanism, we found that miR-142-3p modulated aerobic glycolysis via targeting pyruvate kinase M2 (PKM2). In addition, we demonstrated PKM2 and PKM2-mediated aerobic glycolysis contributes to miR-142-3p-mediated colorectal cancer cell invasion and migration. Hence, these data suggested that miR-142-3p was a potential therapeutic target for the treatment of human colorectal cancer.
\end{abstract}

\section{Introduction}

Colorectal cancer (CRC) is a common neoplasm with an increased rate of morbidity and mortality, and it has become a predominant malignancy worldwide [1]. Although the diagnosis has been improved, the mortality of colorectal cancer is still high.

MicroRNAs (miRNAs) are a group of small noncoding RNAs that play crucial roles in human cancers [2]. Aberrant expression of miRNA is reported to be potential targets for cancer therapy including breast cancer [3], prostate cancer [4], and colorectal cancer [5]. It was reported that miR-142$3 p$ functions as a tumor suppressor and is involved in cancer development. For example, miR-142-3p inhibits the proliferation and invasion of pancreatic cancer cells [6]. A clinical study indicated that miR-142-3p is downregulated in plasma of CRC patients [7]. Moreover, studies suggested miR-142-
$3 p$ is significant CRC progression. Zhu et al. suggested that miR-142-3p suppresses CRC cell growth [8]. Shen et al. indicated that miR-142-3p inhibits CRC cell proliferation via cell cycle regulation [9].

Aerobic glycolysis is a hallmark of cancers [10]. Studies indicated that aerobic glycolysis was involved in cancer cell aggressive behavior $[11,12]$. miRNAs are closely related to aerobic glycolysis. In liver cancer, miR-199a-5p negatively regulates aerobic glycolysis [13]. miR-135 inhibits glycolysis of pancreatic cancer [14]. Accumulating evidence demonstrated that the modulation of aerobic glycolysis by miRNA was achieved by targeting glycolysis-related enzymes including phosphofructokinase-1 (PFK1) [14], pyruvate dehydrogenase kinase 1 (PDK1) [15], hexokinase 2 (HK2) [13], and pyruvate kinase M2 (PKM2) [16]. It has been well characterized that PKM2 plays a significant role in cancer development via aerobic glycolysis [17]. Moreover, previous bioinformatics analysis 
suggested miR-142-3p could directly target PKM2. However, the correlation of miR-142-3p and PKM2 in CRC warrants further investigation.

Here, we showed that miR-142-3p was negative related to colorectal cancer cell invasion and migration. We further demonstrated PKM2, a critical enzyme for glycolytic flux, was a target gene of miR-142-3p. PKM2 and PKM2regulated aerobic glycolysis were required for miR-142-3pmediated cancer progression. Thus, all of these results indicated that miR-142-3p modulates cell invasion and migration via mediating aerobic glycolysis in colorectal cancer.

\section{Materials and Methods}

2.1. Patients and Samples. CRC tissue samples and paired adjacent noncancerous tissue samples that were $2 \mathrm{~cm}$ away from the lesion were collected from 36 patients from the First Affiliated Hospital of Kunming Medical University from March 2016 to January 2018. The age range of patients was 20-60 years old with the average age of $40.36 \pm 11.27$ years old. None of the patients had previously received radiotherapy or chemotherapy before surgery. Written informed consent was received from all participants. The study was approved by the Ethics Committee of Yunnan Cancer Hospital ((2017) Ethics L No. 3).

2.2. Cell Culture and Transfection. Human colonic epithelial cells (NCM460) and human colorectal cancer cells (HCT116 and SW480) were obtained from the American Type Culture Collection (ATCC). Cells were cultured in DMEM supplemented with 10\% heat-inactivated FBS and $100 \mathrm{unit} / \mathrm{mL}$ penicillin-streptomycin at $37^{\circ} \mathrm{C}$ in a $5 \% \mathrm{CO}_{2}$ atmosphere. For cell transfection, miR-142-3p mimic and miR-142-3p NC mimic were constructed by Guangzhou RiboBio Biotechnology Co., Ltd. (Guangzhou, China). $50 \mathrm{nM}$ miR-142-3p NC mimic and 50 nM miR-142-3p mimic were transfected by lipofectamine 2000 transfection reagent (Invitrogen) according to the manufacturer's protocol.

2.3. Western Blotting Assay. Proteins were separated by sodium dodecyl sulfonate polyacrylamide gel electrophoresis (SDS-PAGE) and transferred to the PVDF membrane by wet transfer. After being blocked with 10\% PBS-diluted skim milk, the membrane was incubated with primary antibodies against PKM2 (abcam, ab85555, 1:2000) and GAPDH (abcam, ab9485, 1:5000) at room temperature for 2 hours. After washing 3 times, the membrane was incubated with HRP-conjugated secondary antibodies at room temperature for 1 hour. For the quantification of western blot data, the ImageJ software was used to measure the intensity of each band.

2.4. Quantitative Real-Time PCR (RT-qPCR). The expression of miR-142-3p was detected by qPCR. Tumor tissues were cut into small pieces and grind into powder in liquid nitrogen. The total small RNA extracted from tissues and cancer cells was isolated using TRizol reagent (Invitrogen), and $0.5 \mu \mathrm{g}$ RNA was used as a template for cDNA synthesis using the miRNA 1st Strand cDNA Synthesis Kit (Vazyme, MR101-01). qPCR analysis was performed using 2x SYBR Green PCR Mastermix (Solarbio). qPCR was performed at $95^{\circ} \mathrm{C}$ for $30 \mathrm{~s}, 40$ cycles of $95^{\circ} \mathrm{C}$ for $5 \mathrm{~s}$, and $60^{\circ} \mathrm{C}$ for $60 \mathrm{~s}$. The following primer sequences were used: miR-142-3p forward primer: $5^{\prime}$-TGCGGTGTAGTGTTTCCTACTT-3', reverse primer: $5^{\prime}$-CCAGTGCAGGGTCCGAGGT-3'; U6 forward primer: $5^{\prime}$-CTTCGGCAGCACATATAC- $3^{\prime}$, reverse primer: $5^{\prime}$-GAACGCTTCACGAATTTGC-3'. GAPDH and U6 were used as an internal control. The $2^{-\Delta \Delta \mathrm{Ct}}$ method was used for relative quantification of gene expression.

2.5. Cell Migration and Invasion Assay. Transwell assays were performed using Millipore transwell chambers $(8 \mu \mathrm{m}$ pore size; cat. no. MCEP06H48; Millipore). Transfected HCT116 and SW480 cells $\left(2 \times 10^{4}\right.$ cells/well $)$ were seeded in the upper chambers of a 12-well plate (Corning, Inc.) in a $500 \mu \mathrm{L}$ serum-free medium, and another $500 \mu \mathrm{L}$ complete medium was added to the bottom chamber. The chamber was incubated for $24 \mathrm{~h}$ in a humidified $5 \% \mathrm{CO}_{2}$ incubator at $37^{\circ} \mathrm{C}$. At the end of incubation, cotton-tipped swabs were used to remove the nonmigratory and noninvading cells. For invasion assays, the upper chamber was coated with Matrigel (Millipore) for $30 \mathrm{~min}$ at $37^{\circ} \mathrm{C}$. Then, the remaining cells were fixed with iced formaldehyde for $30 \mathrm{~min}$ and stained using $0.1 \%$ crystal violet at $37^{\circ} \mathrm{C}$ for $1 \mathrm{~h}$. The images were captured using an inverted microscope (Olympus, at 100x magnification) and quantified by Image 1.8 .0 software (National Institutes of Health).

2.6. Dual-Luciferase Reporter Gene Assay. The binding of miR-142-3p and PKM2 was predicted by TargetScan 7.1 (http://www.targetscan.org/vert_71/). The wild-type (WT) or the mutant (MUT) $3^{\prime}$-untranslated region $\left(3^{\prime}\right.$-UTR) of PKM2 containing binding sites of miR-142-3p was cloned into the pmirGLO luciferase reporter vectors (LMAI Bio, LM-1439) using restriction endonucleases. H293T cells were cotransfected with wild-type/mutant-type $3^{\prime}$-UTR and miRNC/miR-142-3p mimic using Lipofectamine 2000 reagents (Invitrogen, 11668019) for $48 \mathrm{~h}$. Cells were lysed, and luciferase activity was assessed.

2.7. Immunohistochemistry. Human colorectal cancer tissues and adjacent tissues were fixed with $4 \%$ paraformaldehyde and dehydrated with ethanol. The samples were embedded in molten paraffin. Paraffin-embedded tissue was cut into sections, and these sections were deparaffinized and heated. Then, tissue sections were incubated with antibodies and detected by the DAB kit (DA1010, Solarbio).

2.8. Enzyme-Linked Immunosorbent Assay. The glucose and lactate levels were measured using a Glucose Assay Kit (K686-100, BioVision) and a Lactic Acid Assay Kit (BC2235, Solarbio), according to the manufacturer's protocol.

2.9. Statistical Analyses. All experiments were carried out at least three times; the representative images were shown. All quantitative data were presented as the mean \pm SEM. Student's $t$-test was used to analyze the differences between the two groups. One-way ANOVA with Tukey's test was used to analyze the differences between multiple groups. $P$ value $<0.05$ was defined as statistically significant. 


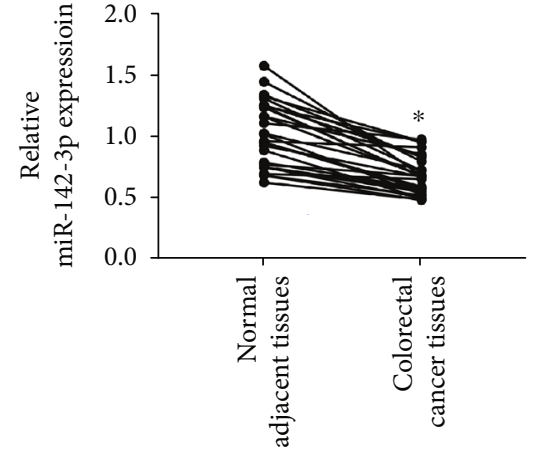

(a)

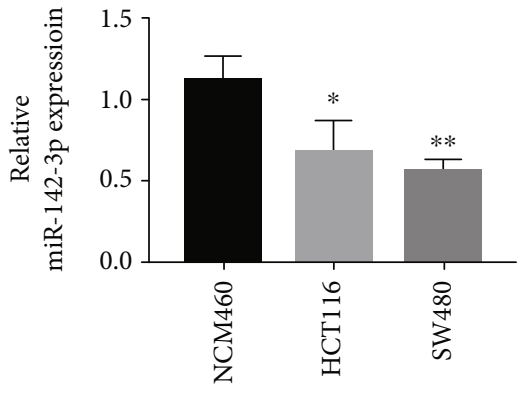

(b)

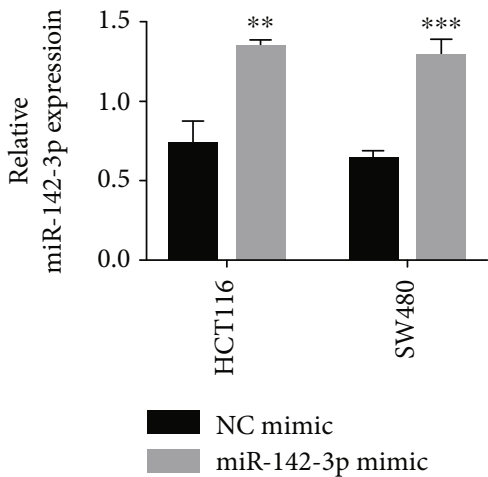

(c)

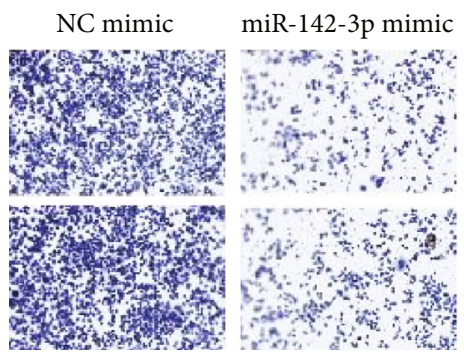

HCT116

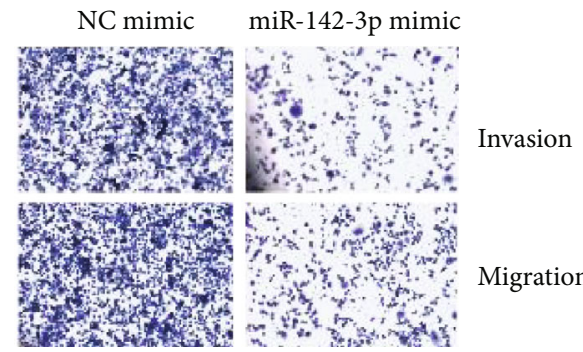

SW480
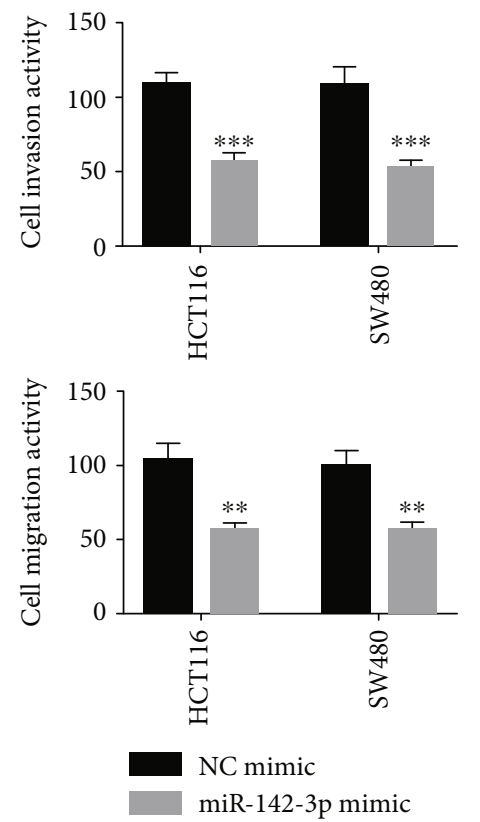

(d)

FIgURE 1: Aberrant expression of miR-142-3p in human colorectal cancer tissues and cell lines. (a) miR-142-3p expression in human colorectal cancer tissues and normal adjacent tissues was determined by RT-qPCR. Data were presented as mean \pm SEM. $P=0.0142$. (b) miR-142-3p expression was determined by RT-qPCR in colorectal cancer cells. Data were presented as mean \pm SEM. $P=0.0344$ and $P=0.0036$. (c) HCT116 and T84 cells were transfected with miR-142-3p mimics and NC mimics. Relative miR-142-3p expressions were determined by RT-qPCR. Data were presented as mean \pm SEM. $P=0.00217$ and $P=0.00082$. (d) Transwell assays were performed to determined invasion and migration of HCT116 and T84 cells. ${ }^{*} P<0.05,{ }^{* *} P<0.01$, and ${ }^{* * *} P<0.001$.

\section{Results}

3.1. miR-142-3p Overexpression Attenuates Cell Invasion and Migration of CRC Cells. In order to determine the role of miR-142-3p in colorectal cancer, we first measured the expression level of miR-142-3p in human colorectal cancer tissues and corresponding normal adjacent tissues with RT-qPCR and observed that miR-142-3p was decreased in colorectal cancer tissues compared with normal adjacent tissues (Figure 1(a)). Moreover, the results of RT-qPCR indicated that the expression level of miR-142-3p in colorectal cancer cell lines was downregulated (Figure $1(\mathrm{~b}),{ }^{*} P<0.05$ and $\left.{ }^{* *} P<0.01\right)$. To further determine the function of miR-142-3p in colorectal cancer, we transfected HCT116 and SW480 cells with miR-142-3p mimics (Figure $1(\mathrm{c}),{ }^{* *} P<0.01$ and $\left.{ }^{* * *} P<0.001\right)$. The results showed that overexpression of
miR-142-3p impaired the invasion and migration in HCT116 and SW480 cells (Figure 1(d), ${ }^{* *} P<0.01$ and ${ }^{* * *} P<0.001$ ). Thus, these data demonstrated that miR-142-3p was downregulated in colorectal cancer and might play a significant role in colorectal cancer development.

3.2. miR-142-3p Directly Targets the $3^{\prime}$-UTR of PKM2. miRNAs usually performing functions by regulating the expression of target genes. According to the TargetScan analysis, there is a binding site for miR-142-3p in the $3^{\prime}$-UTR of PKM2 (Figure 2(a)). To determine whether miR-142-3p could directly target PKM2, we performed dual-luciferase reporter assays. As shown in Figure 2(b), miR-142-3p decreased the luciferase activities of pGL4.49-PKM2-wt but had minimal effect on the luciferase activities of pGL4.49PKM2-mut. In addition, western blot results indicated that 


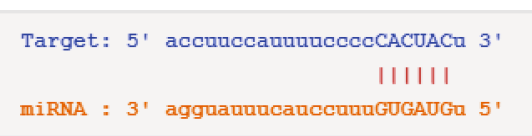

(a)

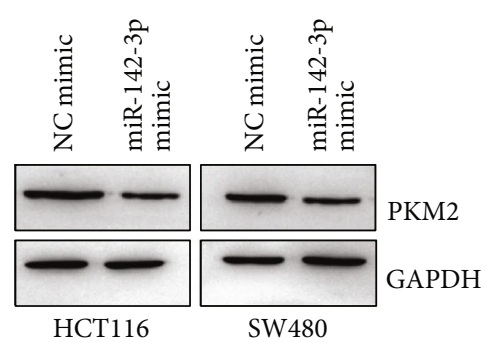

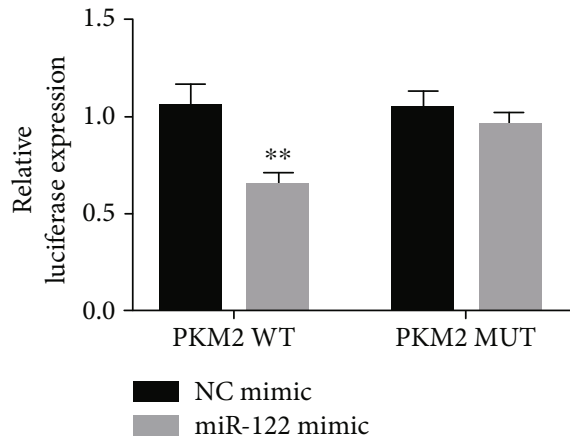

(b)

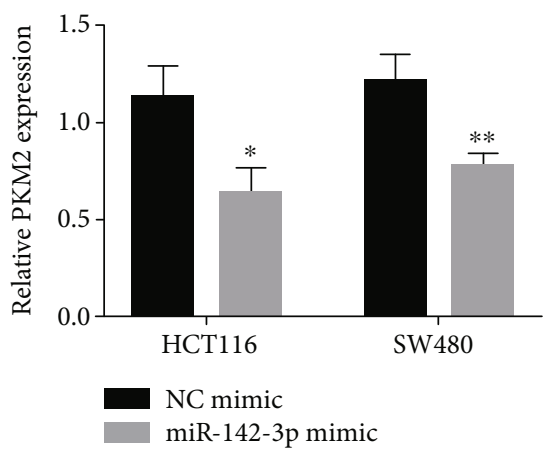

(c)

FIGURE 2: miR-142-3p directly targets the $3^{\prime}$-UTR of PKM2. (a) Binding sites of miR-142-3p and PKM2 were predicted by TargetScan. (b) Dual-luciferase reporter gene assay was performed to measure the relationship between miR-142-3p and PKM2. Data were presented as mean \pm SEM. $P=0.0056$. (c) PKM2 expressions were measured in HCT116 and T84 cells stably overexpressing miR-142-3p. Data were presented as mean \pm SEM. $P=0.0135$ and $P=0.007 .{ }^{*} P<0.05,{ }^{* *} P<0.01$, and ${ }^{* * *} P<0.001$.

overexpression of miR-142-3p inhibited the expression of PKM2 (Figure 2(c)). Collectively, these results showed that miR-142-3p negatively regulated PKM2 via binding to the $3^{\prime}$-UTR of PKM2 mRNA.

3.3. PKM2 Modulates Colorectal Cancer Cell Invasion and Migration via Aerobic Glycolysis Pathway. As an important kinase in the aerobic glycolysis pathway, PKM2 was reported to modulate cancer development via regulating aerobic glycolysis. We first identified PKM2 expression in human CRC via immunohistochemistry and confirmed that PKM2 expression was upregulated in CRC (Figure 3(a)). To validate the role of PKM2 in colorectal cancer development, we overexpressed PKM2 in HCT116 and SW480 cells (Figure 3(b)). The results showed that overexpression of PKM2 resulted in increased cellular glucose uptake and lactate production, which suggested that overexpression of PKM2 in colorectal cancer cells enhanced aerobic glycolysis (Figures 3(c) and $3(d)$ ). In order to identify the role of PKM2 and PKM2mediated aerobic glycolysis in colorectal cancer development, transwell assays were performed. Overexpressed PKM2 promoted invasion and migration of HCT116 and SW480 cells (Figure 3(e)). Thus, these results suggested that PKM2 promoted invasion and migration of HCT116 and SW480 cells via aerobic glycolysis.

3.4. miR-142-3p Regulates Colorectal Cancer Cell Progression via PKM2-Mediated Glycolysis. Based on our previous results, we hypothesized that aberrant expression of miR-142-3p in colorectal cancer might modulate the PKM2-mediated aerobic glycolysis pathway and facilitated cancer development. To test this hypothesis, we transfected NC mimic or miR-142-3p mimic into HCT116 and SW480 cells with PKM2 overexpression (Figure $4(\mathrm{a}),{ }^{*} P<0.05$ and $\left.{ }^{* *} P<0.01\right)$. Overexpression of PKM2 reversed the inhibition effect of miR-142-3p mimic on the invasion and migration in HCT116 and SW480 cells (Figure 4(b), ${ }^{* *} P<0.01$ and $\left.{ }^{* * *} P<0.001\right)$. And then, we focused on the role of miR142-3p in aerobic glycolysis in HCT116 and SW480 cells. As shown in Figures 4(c) and 4(d), miR-142-3p mimic transfection impaired glucose consumption and lactate production. In addition, overexpression of PKM2 reversed the inhibitory effect of miR-142-3p on glucose consumption and lactate production (Figures 4(c) and 4(d), ${ }^{*} P<0.05$ and ${ }^{* *} P<0.01$ ). Thus, these data indicated that miR-142-3p regulated the invasion and migration of colorectal cancer cells via PKM2mediated aerobic glycolysis.

\section{Discussion}

In the present study, we demonstrated that overexpression of miR-142-3p attenuated colorectal cancer cell invasion and migration. About the underlying mechanism, our results indicated that miR-142-3p could directly target $3^{\prime}$-UTR of PKM2 mRNA. PKM2 and PKM2-mediated aerobic glycolysis were required for miR-142-3p overexpression-induced 


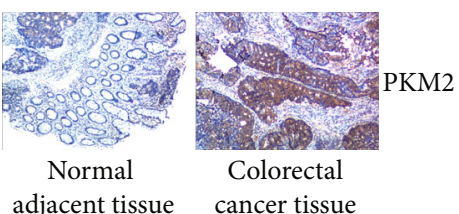

adjacent tissue

cancer tissue

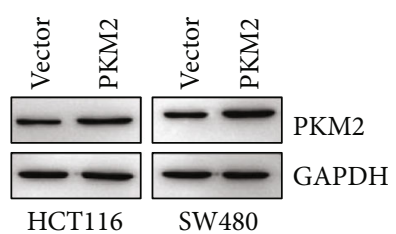

(a)

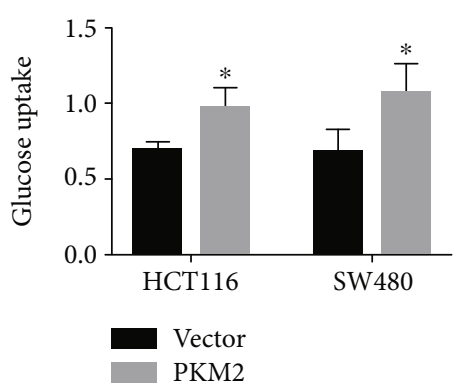

(c)

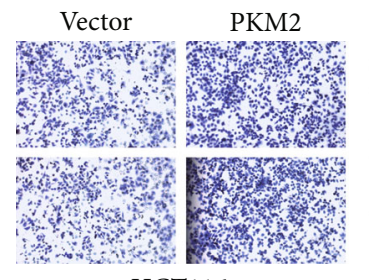

HCT116

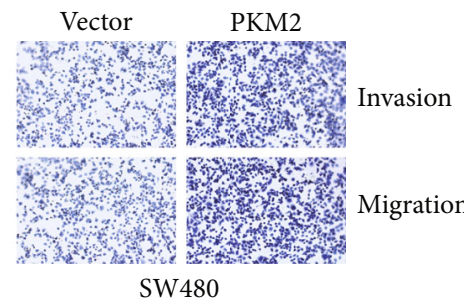

SW480

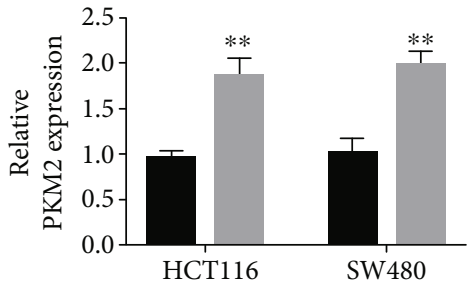

Vector

(b)

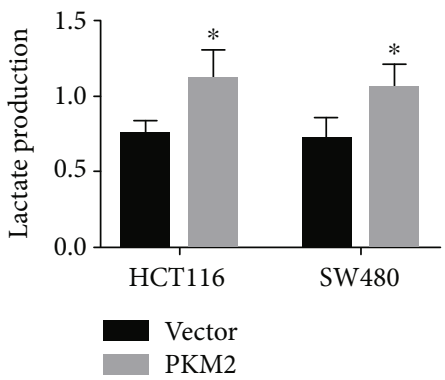

(d)
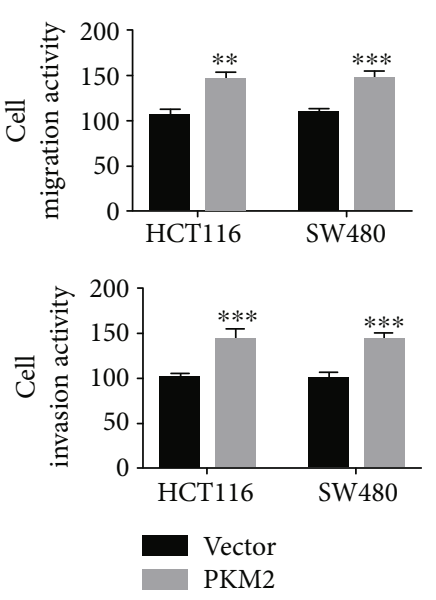

(e)

FIGURE 3: PKM2 modulates colorectal cancer cell invasion and migration via aerobic glycolysis pathway. (a) PKM2 expression in human colorectal cancer tissues and normal adjacent tissues was determined by immunohistochemistry. (b) PKM2 expressions were measured in HCT116 and T84 cells stably overexpressing PKM2. Data were presented as mean \pm SEM. $P=0.002$ and $P=0.0018$. (c) Glucose uptake in HCT116 and T84 cells stably overexpressing PKM2 was measured by ELISA. Data were presented as mean \pm SEM. $P=0.0258$ and $P=0.0422$. (d) Lactate production in HCT116 and T84 cells stably overexpressing PKM2 was measured by ELISA. Data were presented as mean \pm SEM. $P=0.0368$ and $P=0.0452$. (e) Transwell assays were performed to determined invasion and migration of HCT116 and T84 cells stably overexpressing PKM2. ${ }^{*} P<0.05,{ }^{* *} P<0.01$, and ${ }^{* * *} P<0.001$.

inhibition of the invasion and migration in the colorectal cancer cell.

miRNAs are potential therapeutic targets for many cancers including colorectal cancer, and the mechanism of miRNAs involved in cancer progression is different. For example, cancer-associated fibroblast-derived exosomal miRNAs have a great influence on cancer progression [18]. miR-195-5p can modulate M2-like TAM polarization in colorectal cancer [19]. miRNA also involves in inflammation in colorectal cancer [20]. In addition, accumulating evidence indicated that miRNAs played a significant role in cancer cell metabolism. In colorectal cancer, miR-4999-5p reprograms glucose metabolism and affects cell proliferation [21]. miRNAs are reported to alter amino acid metabolism in colorectal cancer [22]. It was reported that miR-142-3p played a crucial role in colorectal cancer. The serum level of miR-142-3p is identified as a prognostic marker in colorectal cancer [23]. And the expression of miR-142-3p is related to CRC detection [24]. In the present study, we found that the level of miR-142-3p is downregulated in CRC tissues and cells. In order to confirm the function of miR-142-3p in colorectal cancer, we first overexpressed miR-142-3p in colorectal cancer cells. We observed overexpression of miR-142-3p attenuated the invasion and migration of colorectal cancer cells, which suggested 

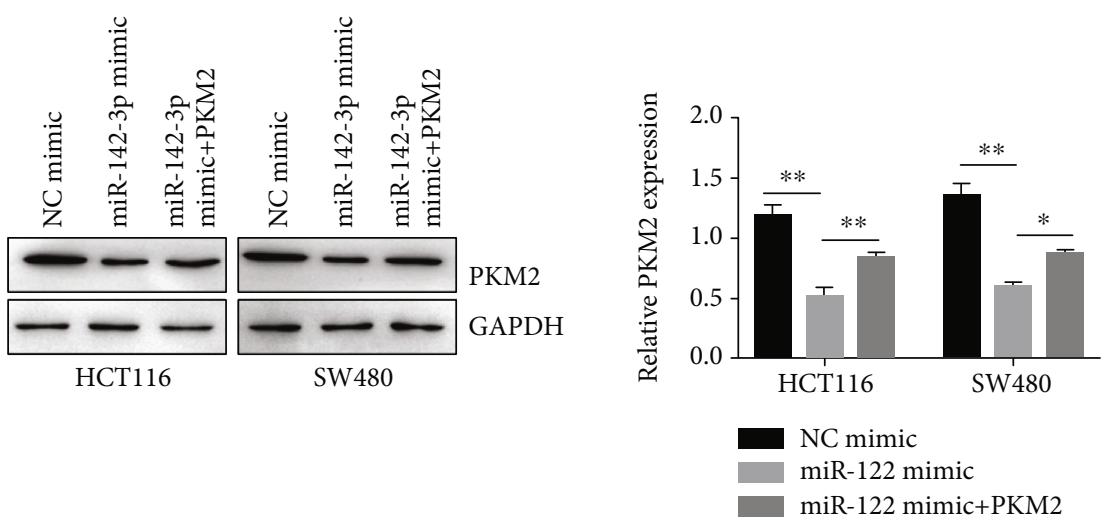

(a)
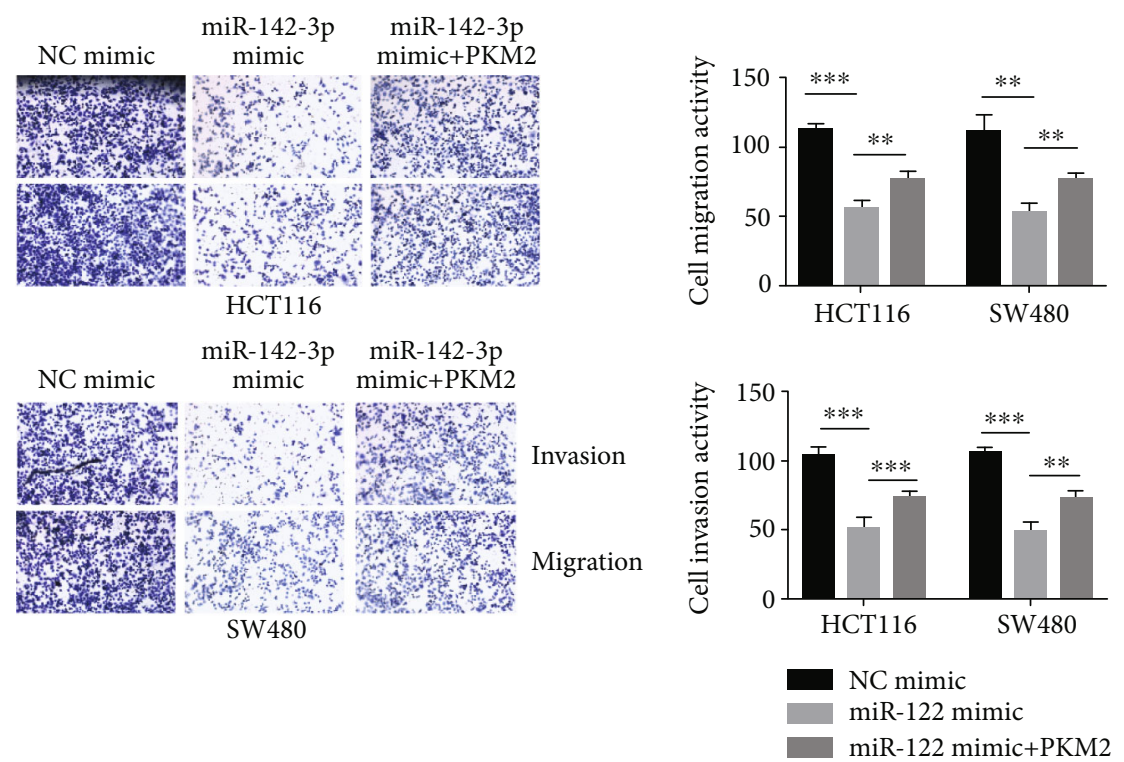

(b)

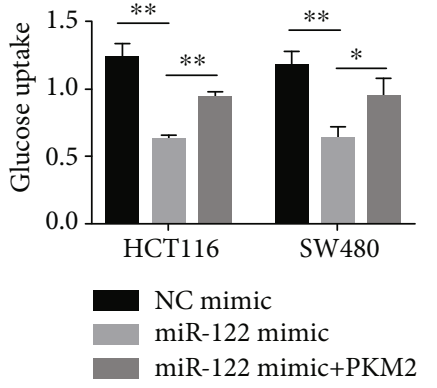

(c)

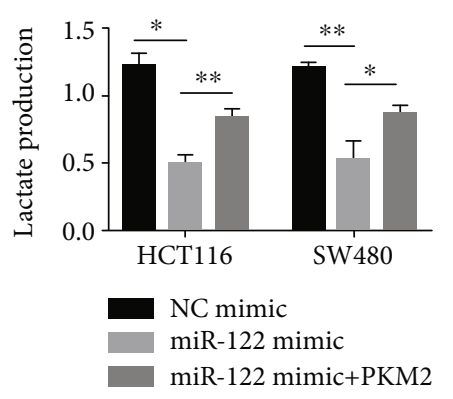

(d)

Figure 4: miR-142-3p regulates colorectal cancer cell progression via PKM2-mediated glycolysis. (a) PKM2 expression was measured in HCT116 and T84 cell stably overexpression miR-142-3p or/and PKM2. Data were presented as mean \pm SEM. $P=0.0065, P=0.002$, $P=0.00745$, and $P=0.0196$. (b) Transwell assays were performed to determined invasion and migration in HCT116 and T84 cell stably overexpression miR-142-3p or/and PKM2. (c) Glucose uptake in HCT116 and T84 cell stably overexpression miR-142-3p or/and PKM2 was measured by ELISA. Data were presented as mean \pm SEM. $P=0.0074, P=0.00558, P=0.00252$, and $P=0.0299$. (d) Lactate production in HCT116 and T84 cell stably overexpression miR-142-3p or/and PKM2 was measured by ELISA. Data were presented as mean \pm SEM. $P=0.047, P=0.0032, P=0.00126$, and $P=0.01839 .{ }^{*} P<0.05,{ }^{* *} P<0.01$, and ${ }^{* * *} P<0.001$.

that miR-142-3p could function as a tumor suppressor for colorectal cancer.

Aerobic glycolysis, a hallmark of cancer cells, has been well highlighted. The regulation of aerobic glycolysis by multiple factors has a strong effect on cancer progression [25]. The role of miR-142-3p in aerobic glycolysis has been well studied [26]. miRNAs usually modulate aerobic glycolysis via targeting related enzymes. For example, miR-142-3p 
suppresses aerobic glycolysis and hepatocellular cancer cell proliferation by targeting LDHA [26]. miR-214 promotes lung cancer cell proliferation and glycolysis by targeting HK2 and PKM2 [27]. It was reported that PKM2 is significant for aerobic glycolysis [28] and PKM2-mediated aerobic glycolysis is closely related to cancer progression [29]. Based on bioinformatics analysis, we found that PKM2 is one of the most significant targets of miR-142-3p. Our study further demonstrated that miR-142-3p could directly target PKM2 in colorectal cancer. Most importantly, PKM2 and PKM2mediated aerobic glycolysis were required for miR-142-3pregulated invasion and migration of colorectal cancer cell. Therefore, our findings indicated that miR-142-3p/PKM2 axis plays a vital role in regulating colorectal cancer metabolism and cancer cell malignancy.

\section{Data Availability}

All data generated or analyzed during this study are included in this published article.

\section{Conflicts of Interest}

The authors declare that they have no competing interests.

\section{Authors' Contributions}

JunYu Ren, Wenliang Li, and Guoqing Pan contributed equally to this work.

\section{References}

[1] P. Wieszczy, M. F. Kaminski, R. Franczyk et al., "Colorectal cancer incidence and mortality after removal of adenomas during screening colonoscopies," Gastroenterology, vol. 158, no. 4, pp. 875-883.e5, 2020.

[2] G. Di Leva, M. Garofalo, and C. M. Croce, "MicroRNAs in cancer," Annual review of pathology, vol. 9, no. 1, pp. 287314, 2014.

[3] J. N. Goh, S. Y. Loo, A. Datta et al., "MicroRNAs in breast cancer: regulatory roles governing the hallmarks of cancer," Biological reviews of the Cambridge Philosophical Society, vol. 91, pp. 409-428, 2016.

[4] L. Schmidt, J. Fredsøe, H. Kristensen et al., "Training and validation of a novel 4-miRNA ratio model ( $\_\mathrm{MiCaP}_{-}$) for prediction of postoperative outcome in prostate cancer patients," Annals of oncology, vol. 29, no. 9, pp. 2003-2009, 2018.

[5] S. Duran-Sanchon, L. Moreno, J. M. Augé et al., "Identification and validation of microRNA profiles in fecal samples for detection of colorectal cancer," Gastroenterology, vol. 158, no. 4, pp. 947-957.e4, 2020.

[6] K. Zhu, Z. Zhang, H. Zhang, Z. Wang, and F. Wang, "MiR$142-3 p$ targeting NUCKS1 inhibits proliferation and invasion of pancreatic cancer cells," Artificial cells, nanomedicine, and biotechnology, vol. 48, no. 1, pp. 415-424, 2020.

[7] R. Ghanbari, N. Mosakhani, J. Asadi et al., "Downregulation of plasma MiR-142-3p and MiR-26a-5p in patients with colorectal carcinoma," Iranian journal of cancer prevention, vol. 8, no. 3, p. e2329, 2015.
[8] X. Zhu, S. Ma, D. Yang et al., "miR-142-3p suppresses cell growth by targeting CDK4 in colorectal cancer," Cellular physiology and biochemistry: international journal of experimental cellular physiology, biochemistry, and pharmacology, vol. 51, no. 4, pp. 1969-1981, 2018.

[9] W. Shen, Z. Zeng, W. Zhu, and G. Fu, "MiR-142-3p functions as a tumor suppressor by targeting CD133, ABCG2, and Lgr5 in colon cancer cells," Journal of molecular medicine (Berlin, Germany), vol. 91, no. 8, pp. 989-1000, 2013.

[10] S. Agnihotri and G. Zadeh, "Metabolic reprogramming in glioblastoma: the influence of cancer metabolism on epigenetics and unanswered questions," Neuro-oncology, vol. 18, no. 2, pp. 160-172, 2016.

[11] Q. Weng, M. Chen, W. Yang et al., "Integrated analyses identify miR-34c-3p/MAGI3 axis for the Warburg metabolism in hepatocellular carcinoma," FASEB journal, vol. 34, no. 4, pp. 5420-5434, 2020.

[12] Y. Zheng, P. Liu, N. Wang et al., "Betulinic acid suppresses breast cancer metastasis by targeting GRP78-mediated glycolysis and ER stress apoptotic pathway," Oxidative medicine and cellular longevity, vol. 2019, Article ID 8781690, 2019.

[13] W. Guo, Z. Qiu, Z. Wang et al., "MiR-199a-5p is negatively associated with malignancies and regulates glycolysis and lactate production by targeting hexokinase 2 in liver cancer," Hepatology, vol. 62, no. 4, pp. 1132-1144, 2015.

[14] Y. Yang, M. B. Ishak Gabra, E. A. Hanse et al., "MiR-135 suppresses glycolysis and promotes pancreatic cancer cell adaptation to metabolic stress by targeting phosphofructokinase-1," Nature communications, vol. 10, 2019.

[15] X. Ma, C. Li, L. Sun et al., "Lin28/let-7 axis regulates aerobic glycolysis and cancer progression via PDK1," Nature communications, vol. 5, no. 1, 2014.

[16] J. Chen, Y. Yu, X. Chen et al., "MiR-139-5p is associated with poor prognosis and regulates glycolysis by repressing PKM2 in gallbladder carcinoma," Cell proliferation, vol. 51, 2018.

[17] H. Zhang, C. Feng, M. Zhang et al., "miR-625-5p/PKM2 negatively regulates melanoma glycolysis state," Journal of cellular biochemistry, vol. 120, no. 3, pp. 2964-2972, 2019.

[18] R. Bhome, R. W. Goh, M. D. Bullock et al., "Exosomal microRNAs derived from colorectal cancer-associated fibroblasts: role in driving cancer progression," Aging, vol. 9, no. 12, pp. 2666-2694, 2017.

[19] X. Lin, S. Wang, M. Sun et al., "miR-195-5p/NOTCH2-mediated EMT modulates IL-4 secretion in colorectal cancer to affect M2-like TAM polarization," Journal of hematology \& oncology, vol. 12, 2019.

[20] C. Josse and V. Bours, "MicroRNAs and inflammation in colorectal cancer," Advances in experimental medicine and biology, vol. 937, pp. 53-69, 2016.

[21] Q. Zhang, Z. Hong, J. Zhu et al., "miR-4999-5p predicts colorectal cancer survival outcome and reprograms glucose metabolism by targeting PRKAA2," OncoTargets and therapy, vol. Volume 13, pp. 1199-1210, 2020.

[22] A. Nijhuis, H. Thompson, J. Adam et al., "Remodelling of microRNAs in colorectal cancer by hypoxia alters metabolism profiles and 5-fluorouracil resistance," Human molecular genetics, vol. 26, no. 8, pp. 1552-1564, 2017.

[23] W. Gao, D. Pang, and S. Yu, "Serum level of miR-142-3p predicts prognostic outcome for colorectal cancer following curative resection," The Journal of International Medical Research, vol. 47 , no. 5, pp. $2116-2125,2019$. 
[24] Z. Kanaan, H. Roberts, M. Eichenberger et al., "A plasma microRNA panel for detection of colorectal adenomas: a step toward more precise screening for colorectal cancer," Annals of Surgery, vol. 258, no. 3, pp. 400-408, 2013.

[25] S. Ganapathy-Kanniappan, "Molecular intricacies of aerobic glycolysis in cancer: current insights into the classic metabolic phenotype," Critical reviews in biochemistry and molecular biology, vol. 53, no. 6, pp. 667-682, 2018.

[26] S. Hua, C. Liu, L. Liu, and D. Wu, "miR-142-3p inhibits aerobic glycolysis and cell proliferation in hepatocellular carcinoma via targeting LDHA," Biochemical and Biophysical Research Communications, vol. 496, no. 3, pp. 947-954, 2018.

[27] K. Zhang, M. Zhang, H. Jiang, F. Liu, H. Liu, and Y. Li, “Downregulation of miR-214 inhibits proliferation and glycolysis in non-small- cell lung cancer cells via down-regulating the expression of hexokinase 2 and pyruvate kinase isozyme M2," Biomedicine \& pharmacotherapy = Biomedecine \& pharmacotherapie, vol. 105, pp. 545-552, 2018.

[28] E. Palsson-McDermott, A. Curtis, G. Goel et al., "Pyruvate kinase M2 regulates Hif- $1 \alpha$ activity and IL- $1 \beta$ induction and is a critical determinant of the Warburg effect in LPSactivated macrophages," Cell Metabolism, vol. 21, no. 1, pp. 65-80, 2015.

[29] T. Li, J. Han, L. Jia, X. Hu, L. Chen, and Y. Wang, "PKM2 coordinates glycolysis with mitochondrial fusion and oxidative phosphorylation," Protein \& Cell, vol. 10, no. 8, pp. 583-594, 2019. 